@CEDA/TU, 2017, US Library of Congress, Catalog Card No.: 79-915209, ISSN: 2091-0339

The Journal of Development and Administrative Studies (JODAS), Vol. 25(1-2), pp. 24-35

\title{
Demographic and Socio-economic Factors Affecting on Health Facility Delivery in Nepal
}

\author{
Sunil Kumar Acharya ${ }^{1}$
}

\begin{abstract}
In Nepal, health facility delivery has improved since the 1990s, but child health delivery facility is still low. In 2001 about 9 percent of the births was delivered at a health facility (MoH, New ERA, and ORC Macro, 2002) which increased to 35 percent in 2011 (MOHP, New ERA, and ICF International Inc, 2012) and further increased to 57 percent in 2016 (MoH, New ERA and ICF, various 2017). This is a rapid increase in health facility delivery since 1996 but still nearly one-half of delivery take place at home. Research in developing countries shows that demographic, social and economic factors influence the utilization of health facility for delivery of births. This paper examines the likelihood of health facility delivery in relation to women's demographic, social and economic status in Nepal. The 2011 Nepal Demographic and Health Survey (NDHS) data set has been utilized by applying bivariate logistics regression analysis technique to examine the effects of these variables in health facility delivery in Nepal. The analysis findings show large variations and gaps on delivery care based on demographic, social and economic status of women. Against this finding, the study concludes that there is a need for the implementation of appropriate policy and program measures by the government and other agencies to address the existing variations and gaps in utilization of health facility for delivery of births among different sub-groups of women in Nepal. Further research studies focusing on the existing barriers in health facility delivery need to be conducted in Nepal especially among women who are disadvantaged and marginalized.
\end{abstract}

Key words: Maternal mortality, delivery practice, skilled birth attendance

\section{Introduction}

In 1996, the Nepal Family Health Survey (NFHS) estimated Nepal's MMR at 539 per 100,000 live births, the highest in South Asia. The Demographic and Health Survey conducted in 2006 estimated MMR to be 281 which was much reduced but still one of the higher MMRs globally (MoHP, New ERA \& Macro International Inc., 2007). The Maternal Mortality and Morbidity study conducted in Nepal in 2008 in eight of the 75 districts of the country found MMR to be 229 per 100,000 live births, ranging from a MMR of 153 in Okhaldhunga district to 301 in Rasuwa (Suvedi et al,). The study also showed considerable variation in MMR between women of various ages and caste/ethnic groups. The most recent estimates for Nepal done by NDHS 2016 is 239 deaths per 100000 live births which is slightly higher than the previous estimates of MMR by 2008 study. In 2016 Forty-three percent of deliveries took place in government facilities and 10 percent in private facilities. The most frequently reported reason for not delivering births at health facility was: not necessary to deliver in a health facility, birth takes place before reaching the facility, the facility being too far and not having transport. Notably, 80 percent of mothers in Province 2 said they felt that it was not necessary to

\footnotetext{
${ }^{1}$ Dr. Acharya is a Lecturer at Central Department of Population Studies, Tribhuvan University, Kathmandu, Nepal

Corresponding mail: sunilacharya880@gmail.com
} 
deliver in a health facility. Underutilization of safe motherhood especially health facility delivery services is considered as the main factor contributing to high maternal mortality, since nearly one-half of births still take place at home, many without skilled health providers, utilization of ANC services is low and the trend of birth preparation is still low (MOHP, New ERA, and ICF International Inc, 2012).

Every year, more than 500,000 maternal deaths occur worldwide, 4 million newborns die and another 3 million babies are stillborn. Nearly all these deaths take place in low- and middle-income countries and most could be prevented with current medical care (WHO, 2012). Most obstetric complications occur around the time of delivery and cannot be predicted. Therefore it is important that all pregnant women have access to a health facility or a skilled birth attendant, i.e. someone with midwifery skills, who is able to manage a normal delivery and who can recognize and manage obstetric complications, or refer in time if needed. Health facility delivery is advocated as the single most important factor in preventing maternal deaths.

The paper is divided into five sections. The first section presents background to current trend of maternal mortality and health facility delivery practices of women in Nepal. Second section provides a brief literature review. Third section describes methodology adopted in detail including type of data and analysis techniques. Section Four presents analysis and interpretation of the findings. Section five presents discussion and conclusion of the research analysis.

\section{Literature Review}

Health facility delivery or skilled birth attendance at delivery is determined by a number of factors including a woman's location, socio-economic status and education, cultural and religious beliefs. A woman's decision-making power within the family, and her past experiences in childbearing may also affect the care she receives. The importance of these factors may differ from setting to setting within a country. National trends provided by large-scale surveys can mask substantial variations among different groups of women, especially the inequities between rich and poor. A study of trends across six developing countries - Bangladesh, Bolivia, Ghana, Indonesia, Malawi and the Philippines found correlations between these factors and skilled birth attendance. Some of these factors include, urban-rural residence, economic status, maternal education, and practice of ANC and birth order (UNFPA, 2004).

Studies conducted in other countries including Ethiopia (Teferra, Alemu \& Woldeyohannes, 2012; Tsegay, Tesfay, Goicolea, Edin, Lemma \& Sebastian, 2013; Mehari, 2013), Uttar Pradesh/India (Bloom and Lippeveld, 1999), Andhra Pradesh/India (Nair, Ariana \& Webster, 2012) and Nepal (Dahal, 2013; Shah, Rehfuess, Maskey. Fischer, Bhandari \& Delius, 2015) have found strong association between background of women (social, cultural, economic, and demographic) and health facility delivery or skilled birth attendance.

Few research studies have been conducted in Nepal on aspects of delivery care including demographic and socio-economic factors affecting health facility utilization for delivery by women. The finding of most of these studies is limited either to specific geographical locations, communities or only a few districts. These studies also have not included a spectrum of factors that may influence the delivery service utilization behavior of women in Nepal. There is a need therefore to explore and identify the variables at the national and regional level that have either facilitated or hindered in accessing health facility delivery of births. The analysis result could be expected to fill the information gaps and also provide inputs for future policy and programs.

Thus this paper sought answer to following research question: what are the demographic, social and economic factors that may affect health facility delivery of births in Nepal? The analysis result has shed light on the factors associated with utilization or non-utilization of health facility for delivery by women in Nepal. The paper also presents the analysis by rural and urban residence and by 3 ecological belts (Mountain, Hill and Tarai) of Nepal. 


\section{Methodology}

Data files of Nepal Demographic and Health Survey 2011 (NDHS, 2011) have been utilized to analyze the effects of demographic, social and economic factors on the utilization of health facility delivery services in Nepal. This is a nationally representative survey and the total sample consists of 4,079 currently married women between the ages of 15-49 years who had given birth to a child within the 5 years preceding the survey date. After applying weight age the sample size is derived at 4,148 women.

The paper conceptualizes that there are specific categories and number of variables that determine the decision making processes of a woman or her family members on whether to deliver the birth at health facility or not. Thus the paper has defined two sets of variables which are respectively categorized as dependent and independent. Health facility delivery is taken as dependent variable. Independent variables include demographic (woman's current age, age at $1^{\text {st }}$ birth, children ever born, number of children <5 years of age, pregnancy termination (induced abortion); social (castelethnicity, religion, woman's education, husband's education, mass media habits, spatial distribution, and rural/urban residence); and economic (woman's occupation, husband's occupation, woman's employment status, household wealth) concerning the woman and her family members. These variables influence the delivery behavior and practices of an individual woman.

In data analysis the dependent and independent variables have been re-coded and specific variables have been re-grouped from the data file to make a meaningful analysis. Data have been analyzed by using STATA software. First, data are analyzed on the basis of cross tabulations of dependent and independent variables. Second, bivariate logistic regression is used to predict the likelihood of utilization of health facility delivery services by women in Nepal.

\section{Analysis of Results}

\subsection{Demographic factors and utilization of health facility delivery in Nepal}

In Nepal the trend of child delivery at a hospital or other health facilities where skilled birth attendants are available is low. In 2011 only about 35 percent of births took place in a health facility: 26 percent were delivered in a public-sector health facility, 2 percent in a nongovernment facility, and 7 percent in private facility. Nearly two-thirds of the births (63 percent) were taking place at home (MOHP, New ERA, and ICF International Inc, 2012).

The NDHS results show that by age majority of the young women especially those in age groups of 15-19 (54.4\%), 20-24 (42.1\%) and 25-29 (40.2\%) had delivered at a health facility. Similarly, higher percentage of women whose age at first birth was in the age group of 25-29 (58.7\%) and 20-24 (43.3\%) were the ones who delivered at a health facility. Similarly comparatively higher percentage of women who had low parity $1(60.1 \%)$ and $2(39.7 \%)$ had delivered at a health facility than the women who had 3 or more children. Comparatively low percentage of women with high parity $(3,4$, 5 or more number of children) had delivered their last child at a health facility.

Similarly women who had fewer number of under 5 year aged children at the time of the survey had delivered at a health facility than the women with higher number of under 5 children. About 42 percent of women who had a history of pregnancy termination had also made health facility delivery of their last child. Health facility delivery practice was also found high among women (58\%) who had made ANC visits 4 or more times in the last pregnancy (Table 1). 


\begin{tabular}{|c|c|c|c|c|c|c|}
\hline \multirow{2}{*}{\begin{tabular}{l|} 
Description \\
Age group \\
\end{tabular}} & \multicolumn{2}{|c|}{$\begin{array}{c}\text { Health facility } \\
\text { delivery }\end{array}$} & \multicolumn{2}{|c|}{$\begin{array}{c}\begin{array}{c}\text { Delivery at home or } \\
\text { other places }\end{array} \\
\end{array}$} & \multicolumn{2}{|c|}{ Total respondents } \\
\hline & Number & Percent & Number & Percent & Number & Percent \\
\hline $15-19$ & 181 & 54.4 & 148 & 44.4 & 333 & 100.0 \\
\hline $20-24$ & 559 & 42.1 & 744 & 56.0 & 1328 & 100.0 \\
\hline $25-29$ & 526 & 40.2 & 767 & 58.5 & 1310 & 100.0 \\
\hline $30-34$ & 226 & 33.7 & 433 & 64.6 & 670 & 100.0 \\
\hline $35-39$ & 76 & 24.0 & 235 & 74.1 & 317 & 100.0 \\
\hline $40-44$ & 29 & 20.7 & 108 & 77.1 & 140 & 100.0 \\
\hline $45-49$ & 1 & 2.0 & 45 & 90.0 & 50 & 100.0 \\
\hline Total & 1598 & 38.5 & 2480 & 59.8 & 4148 & 100.0 \\
\hline \multicolumn{7}{|l|}{ Age at first birth* } \\
\hline $10-14$ & 14 & 21.5 & 51 & 78.5 & 65 & 100.0 \\
\hline $15-19$ & 688 & 32.4 & 1407 & 66.2 & 2125 & 100.0 \\
\hline $20-24$ & 712 & 43.3 & 902 & 54.8 & 1645 & 100.0 \\
\hline $25-29$ & 155 & 58.7 & 100 & 37.9 & 264 & 100.0 \\
\hline $30+$ & 29 & 60.4 & 19 & 39.6 & 48 & 100.0 \\
\hline Total & 1598 & 38.5 & 2479 & 59.8 & 4147 & 100.0 \\
\hline \multicolumn{7}{|c|}{ Children ever born* } \\
\hline 1 & 782 & 60.1 & 500 & 38.4 & 1302 & 100.0 \\
\hline 2 & 461 & 39.7 & 682 & 58.7 & 1162 & 100.0 \\
\hline 3 & 197 & 26.9 & 523 & 71.4 & 733 & 100.0 \\
\hline 4 & 93 & 23.4 & 296 & 74.6 & 397 & 100.0 \\
\hline $5+$ & 65 & 11.7 & 479 & 86.2 & 556 & 100.0 \\
\hline Total & 1598 & 38.5 & 2480 & 59.8 & 4150 & 100.0 \\
\hline \multicolumn{7}{|l|}{ Child < 5 years* } \\
\hline None & 120 & 48.8 & 117 & 47.6 & 246 & 100.0 \\
\hline 1 & 976 & 44.8 & 1172 & 53.7 & 2181 & 100.0 \\
\hline 2 & 412 & 30.9 & 902 & 67.6 & 1335 & 100.0 \\
\hline 3 & 65 & 21.7 & 228 & 76.0 & 300 & 100.0 \\
\hline $4+$ & 25 & 29.1 & 60 & 69.8 & 86 & 100.0 \\
\hline \multicolumn{7}{|l|}{$\begin{array}{l}\text { Pregnancy } \\
\text { Termination* }\end{array}$} \\
\hline Yes & 331 & 41.5 & 451 & 56.6 & 797 & 100.0 \\
\hline No & 1267 & 37.8 & 2029 & 60.5 & 3352 & 100.0 \\
\hline \multicolumn{7}{|c|}{$\begin{array}{l}\text { Utilization of ANC } 4 \\
\text { or more }\end{array}$} \\
\hline Yes & 1205 & 58.0 & 841 & 40.5 & 2079 & 100.0 \\
\hline No & 393 & 19.0 & 1639 & 79.1 & 2071 & 100.0 \\
\hline Total & 1598 & 38.5 & 2480 & $\begin{array}{l}59.8 \\
\end{array}$ & 4150 & 100.0 \\
\hline \multicolumn{7}{|c|}{$\begin{array}{l}\text { Source: Calculated from NDHS } 2011 \text { data files } \\
\text { Weights are applied } \\
\text { *71 weighted cases are missing }\end{array}$} \\
\hline
\end{tabular}

The overall logistic regression result derived from NDHS 2011 data shows that women of younger ages are highly likely to opt for health facility delivery than older women. Women who are aged 1519 and 20-24 years respectively are 64 times (95\% CI 14.523-282.970) and 39 times (95\% CI 9.087170.440) more likely to have health facility delivery than women who are at 30 years or higher ages. The results are significant at $(\mathrm{p}<0.001)$ level (Table 2$)$. 


\begin{tabular}{|c|c|c|c|}
\hline Variable name & Odds ratio (OR) & $\begin{array}{c}\text { Confidence interval } \\
\text { (CI) }\end{array}$ & $P$ value \\
\hline \multicolumn{4}{|l|}{ Age group } \\
\hline $15-19$ & 64.105 & $14.523-282.970$ & 0.000 \\
\hline $20-24$ & 39.355 & $9.087-170.440$ & 0.000 \\
\hline $25-29$ & 35.953 & $8.304-155.682$ & 0.000 \\
\hline $30-34$ & 27.413 & $6.288-119.517$ & 0.000 \\
\hline $35-39$ & 16.921 & $3.802-75.315$ & 0.000 \\
\hline $40-44$ & 13.869 & $2.926-65.739$ & 0.001 \\
\hline $45-49$ & Ref. & & \\
\hline \multicolumn{4}{|l|}{ Children Ever Born } \\
\hline 1 & 11.580 & $8.136-16.482$ & 0.000 \\
\hline 2 & 4.992 & $3.501-7.118$ & 0.000 \\
\hline 3 & 2.787 & $1.902-4.084$ & 0.000 \\
\hline 4 & 2.313 & $1.508-3.548$ & 0.000 \\
\hline $5+$ & Ref. & & \\
\hline \multicolumn{4}{|l|}{ Age at first birth } \\
\hline $10-14$ & .5542 & $.251-1.223$ & 0.144 \\
\hline $15-19$ & Ref. & & \\
\hline $20-24$ & 1.612 & $1.370-1.898$ & 0.000 \\
\hline $25-29$ & 3.168 & $2.295-4.371$ & 0.000 \\
\hline $30+$ & 3.076 & $1.470-6.436$ & 0.003 \\
\hline \multicolumn{4}{|l|}{ Child $<5$ years } \\
\hline 0 & 2.468 & $1.272-4.786$ & 0.008 \\
\hline 1 & 2.005 & $1.112-3.618$ & 0.021 \\
\hline 2 & 1.099 & $.605-1.999$ & 0.756 \\
\hline 3 & .684 & $.351-1.335$ & 0.266 \\
\hline $4+$ & Ref. & & \\
\hline \multicolumn{4}{|l|}{ Pregnancy termination } \\
\hline Yes & 1.177 & $.974-1.421$ & 0.091 \\
\hline No & Ref. & & \\
\hline \multicolumn{4}{|c|}{ Utilization of ANC 4 or more } \\
\hline Yes & 5.975 & $5.189-6.880$ & 0.000 \\
\hline No & Ref. & & \\
\hline \multicolumn{4}{|c|}{$\begin{array}{l}\text { Source: Calculated from NDHS } 2011 \text { data files } \\
\text { Weights are applied } \\
\text { Ref: Reference category }\end{array}$} \\
\hline
\end{tabular}

The analysis results further show that low parity women, those who have 1 child and women who have 2 children respectively are 11 times (95\% CI 8.136-16.482) and 4 times (95\% CI 3.501=7.118) more likely to deliver their pregnancy at a health institution. Similarly, women who have first birth in their twenties (20-24 and 25-29 age groups) are highly likely to deliver in a health institution than those who have first birth at 19 years or at lower ages. Similarly, the logistic regression analysis results further show that women who have 1-2 children under five years of age are also twice as likely $(\mathrm{p}<0.01)$ to have health facility delivery than women with 3 or more under 5 children. Similarly, women who have a history of pregnancy termination (induced abortion) are also more likely have health facility delivery. The study result further showed that the odds of women who have taken ANC services 4 or more times of having health facility delivery is nearly 6 times more than women who had taken ANC services less than 4 times (95\% CI 5.189 - 6.880; $\mathrm{p}<0.001$ ) (Table 2).

\subsection{Social factors and utilization of health facility delivery in Nepal}

The 2011 NDHS results show that a high percentage of women (71.1\%) from Newar groups had delivered at a health institution. Similarly, relatively high percentage of women (48\%) from 
Brahmin/Chhetri caste groups, women from 'Other caste groups' (38.4\%) and women from Muslim groups (37.3\%) had delivered in a health institution compared to women from other caste/ethnic groups. The percentage of women from Hill and Tarai Janjati groups (31.1\%) and Dalit groups (29.7\%) was slightly lower who had delivered their last pregnancy at a health institution (Table 3).

\begin{tabular}{|c|c|c|c|c|c|c|}
\hline \multirow{2}{*}{ Description } & \multicolumn{2}{|c|}{ Health facility delivery } & \multicolumn{2}{|c|}{$\begin{array}{c}\text { Delivery at home or } \\
\text { other places }\end{array}$} & \multicolumn{2}{|c|}{ Total respondents } \\
\hline & Number & Percent & Number & Percent & Number & Percent \\
\hline \multicolumn{7}{|l|}{ Caste/ethnicity+ } \\
\hline $\begin{array}{l}\text { Brahmin/Chhetri \& other } \\
\text { castes }\end{array}$ & 616 & 48.0 & 649 & 50.6 & 1283 & 100.0 \\
\hline Other Tarai Caste & 159 & 38.4 & 250 & 60.4 & 414 & 100.0 \\
\hline Dalit & 203 & 29.7 & 470 & 68.8 & 683 & 100.0 \\
\hline Newar & 91 & 71.1 & 35 & 27.3 & 128 & 100.0 \\
\hline Hill and Tarai Janajati & 434 & 31.1 & 926 & 66.3 & 1396 & 100.0 \\
\hline Muslim & 88 & 37.3 & 148 & 62.7 & 236 & 100.0 \\
\hline \multicolumn{7}{|l|}{ Religion* } \\
\hline Hindu & 1370 & 39.8 & 2009 & 58.3 & 3445 & 100.0 \\
\hline Others & 227 & 32.3 & 471 & 67.0 & 703 & 100.0 \\
\hline \multicolumn{7}{|l|}{ Residence* } \\
\hline Rural & 1286 & 34.5 & 2377 & 63.7 & 3730 & 100.0 \\
\hline Urban & 311 & 74.4 & 103 & 24.6 & 418 & 100.0 \\
\hline \multicolumn{7}{|l|}{ Ecological Region* } \\
\hline Mountain & 64 & 20.9 & 236 & 77.1 & 64 & 100.0 \\
\hline Hill & 580 & 34.8 & 1047 & 62.8 & 580 & 100.0 \\
\hline Tarai & 953 & 43.8 & 1197 & 55.1 & 953 & 100.0 \\
\hline \multicolumn{7}{|l|}{$\begin{array}{l}\text { Respondent's } \\
\text { Education* }\end{array}$} \\
\hline Higher & 216 & 82.1 & 41 & 15.6 & 263 & 100.0 \\
\hline Secondary & 719 & 58.5 & 490 & 39.9 & 1229 & 100.0 \\
\hline Primary & 285 & 34.2 & 531 & 63.7 & 834 & 100.0 \\
\hline No education & 377 & 20.7 & 1418 & 77.8 & 1822 & 100.0 \\
\hline \multicolumn{7}{|l|}{ Husband's Education* } \\
\hline Higher & 335 & 72.7 & 118 & 25.6 & 461 & 100.0 \\
\hline Secondary & 829 & 45.9 & 953 & 52.7 & 1808 & 100.0 \\
\hline Primary & 268 & 27.2 & 698 & 70.9 & 984 & 100.0 \\
\hline No education & 162 & 18.6 & 691 & 79.3 & 871 & 100.0 \\
\hline \multicolumn{7}{|l|}{ Media Habits } \\
\hline \multicolumn{7}{|l|}{ Newspaper } \\
\hline At least once a week & 247 & 82.6 & 48 & 16.1 & 299 & 100.0 \\
\hline Less than once a week & 448 & 57.6 & 317 & 40.7 & 778 & 100.0 \\
\hline Not at all & 902 & 29.4 & 2115 & 68.9 & 3071 & 100.0 \\
\hline \multicolumn{7}{|l|}{ Radio* } \\
\hline At least once a week & 695 & 45.2 & 818 & 53.3 & 1536 & 100.0 \\
\hline Less than once a week & 565 & 34.9 & 1020 & 63.1 & 1617 & 100.0 \\
\hline Not at all & 338 & 33.9 & 642 & 64.5 & 996 & 100.0 \\
\hline \multicolumn{7}{|l|}{ Television* } \\
\hline At least once a week & 969 & 62.1 & 573 & 36.7 & 1561 & 100.0 \\
\hline Less than once a week & 354 & 30.9 & 767 & 67.0 & 1144 & 100.0 \\
\hline Not at all & 274 & 19.0 & 1140 & 79.1 & 1442 & 100.0 \\
\hline \multicolumn{7}{|l|}{ Mass Media Habits* } \\
\hline Yes & 130 & 80.2 & 30 & 18.5 & 162 & 100.0 \\
\hline No & 1467 & 36.8 & 2450 & 61.5 & 3986 & 100.0 \\
\hline Total & 1597 & 38.5 & 2480 & 59.8 & 4148 & 100.0 \\
\hline \multicolumn{7}{|c|}{$\begin{array}{l}\text { Source: Calculated from NDHS } 2011 \text { data files } \\
\text { Weights are applied } \\
\text { Totals may not equal } 100.0 \text { due to rounding off of cases } \\
\text { +List of caste/ethnic groupings is attached as Annex } 1 \\
\text { *71 weighted cases are missing }\end{array}$} \\
\hline
\end{tabular}


By religion, higher percentage of women following Hinduism (39.8\%) had their last pregnancy delivered at a health institution. Comparatively lower percentage of women following other religions (such as Buddhism, Islam, Kirat, Christianity and others) had delivered their last child at a health facility or health institution. The 2011 NDHS data also reveal some variation in health facility delivery practices of women by their current place of residence. For example, a high percentage of woman (74.4\%) residing in urban areas had opted for health facility delivery of their last pregnancy. Comparatively a low percentage of women i.e., 35 percent (about one-third of all women) had delivered their last child in a health facility (Table 3).

By ecological distribution, highest percentage of health facility delivery was found in Tarai $(43.8 \%)$ followed by women living in Hill (34.8\%) areas. The lowest percentages of women having health facility delivery were from the Mountain (20.9\%) areas (Table 3).

By education, more than 8 in every 10 women $(82.1 \%$; $n=216)$ with higher level of education (higher secondary and university) had their last pregnancy at a health institution. Similarly more than one-half of women with secondary level of education $(58.5 \%)$ had health facility delivery of last pregnancy. Slightly over one-third of women (34.2\%) with primary level of education also had health facility delivery of last child. Similarly, a high percentage of women (72.7\%) whose husband had attained higher level of education had also delivered last child at health institution. The NDHS results further show that about 80 percent of women who have access to mass media have had their last child delivered at a health institution (Table 3).

Studies conducted in Nepal and other developing countries have clearly exhibited the influence of social variables on use or non-use of health institutions for child delivery (Nzioki, Onyango \& Ombaka, 2015; Dahal, 2013; Shah et al., 2015; and Dhak, 2013). Social characteristics like women's education and employment status, husband education, ethnicity, place of residence are shown to have determining effect in health facility delivery practices. The effect of social variables on health facility delivery practices of Nepalese women is presented in Table 4. By caste ethnicity, the analysis results show that among the different caste/ethnic groups in Nepal Newar women are more than two times likely to have health facility delivery (OR 2.73; 95 CI 1.663-4.496) than women from other caste/ethnic groups. Similarly, Hindu women (OR 1.41; 95 CI 1.132-1.763) are also more likely to have health facility delivery than non-Hindu women. By place of residence women living in urban areas are more than 5 times likely (OR 5.56; 95 CI 4.633-6.768) to have health facility delivery than women living in rural areas (Table 4).

Research conducted in Nepal and elsewhere shows that educational attainment of women has strong effect on health services seeking behavior. The analysis of NDHS 2011 data also confirms this finding. Women who have attained higher education are strongly likely (OR 20.05; 95 CI 12.77731.464) to have health facility delivery than the women who have no education or have only primary or secondary level of education. Husband education, however, does not show much effect on the decision to have health facility delivery (Table 4 ).

Regarding access to media channels, the analysis results show that access to newspaper has much higher likelihood effect on health facility delivery practices than access to other media channels like Radio and TV. Women with access to newspaper at least once a week have 11 times more likelihood of health facility delivery (95\% CI 8.360 - 15.488; p<.001) than women who have access less than once a week or no access. Access to radio also has some likelihood effect of health facility delivery as women with access to radio at least once a week are 1.06 times likely (95\% CI $1.363-1.897 ; \mathrm{p}<.001$ ) than the women who have limited 


\begin{tabular}{|c|c|c|c|}
\hline Description & $\begin{array}{l}\text { Odds ratio } \\
\text { (OR) }\end{array}$ & $\begin{array}{l}\text { Confidence } \\
\text { interval } \\
(95 \text { CI }) \\
\end{array}$ & $P$ value \\
\hline \multicolumn{4}{|l|}{ Caste/ethnicity* } \\
\hline Brahmin/Chhetri \& other castes & Ref. & & \\
\hline Other Tarai Caste & .668 & $.493-.903$ & 0.009 \\
\hline Dalit & .454 & $.360-.574$ & 0.000 \\
\hline Newar & 2.734 & $1.663-4.496$ & 0.000 \\
\hline Hill and Tarai Janajati & .494 & $.411-.593$ & 0.000 \\
\hline Muslim & .623 & $.416-.933$ & 0.022 \\
\hline \multicolumn{4}{|l|}{ Religion } \\
\hline Hindu & 1.412 & $1.132-1.763$ & 0.002 \\
\hline Others & Ref. & & \\
\hline \multicolumn{4}{|l|}{ Residence } \\
\hline Rural & Ref. & & \\
\hline Urban & 5.560 & $4.633-6.768$ & 0.000 \\
\hline \multicolumn{4}{|l|}{ Ecological Region } \\
\hline Mountain & .340302 & $.275-.422$ & 0.000 \\
\hline Hill & .6956638 & $.592-.817$ & 0.000 \\
\hline Tarai & Ref. & & \\
\hline \multicolumn{4}{|l|}{ Respondent's Education } \\
\hline Higher & 20.050 & $12.777-31.464$ & 0.000 \\
\hline Secondary & 5.519 & $4.516-6.745$ & 0.000 \\
\hline Primary & 2.020 & $1.612-2.532$ & 0.000 \\
\hline No education & Ref. & & \\
\hline \multicolumn{4}{|l|}{ Husband's Education } \\
\hline Higher & .305 & $.234-.399$ & 0.000 \\
\hline Secondary & .135 & $.100-.181$ & 0.000 \\
\hline Primary & .082 & $.059-.115$ & 0.000 \\
\hline No education & Ref. & & \\
\hline \multicolumn{4}{|l|}{ Media Habits } \\
\hline \multicolumn{4}{|l|}{ Newspaper } \\
\hline At least once a week & 11.379 & $8.360-15.488$ & 0.000 \\
\hline Less than once a week & 3.263 & $2.775-3.837$ & 0.000 \\
\hline Not at all & Ref. & & \\
\hline \multicolumn{4}{|l|}{ Radio } \\
\hline At least once a week & 1.608 & $1.363-1.897$ & 0.000 \\
\hline Less than once a week & 1.046 & $.885-1.235$ & .599 \\
\hline Not at all & Ref. & & \\
\hline \multicolumn{4}{|l|}{ Television } \\
\hline At least once a week & 6.973 & $5.903-8.236$ & 0.000 \\
\hline Less than once a week & 1.905 & $1.588-2.284$ & 0.000 \\
\hline Not at all & Ref. & & \\
\hline \multicolumn{4}{|l|}{ Mass Media Habits } \\
\hline Yes & 7.293 & $4.334-12.273$ & 0.000 \\
\hline No & Ref. & & \\
\hline \multicolumn{4}{|c|}{$\begin{array}{l}\text { Source: Calculated from NDHS } 2011 \text { data files } \\
\text { Weights are applied } \\
\text { *List of caste/ethnic groupings is attached as Annex } 1 \\
\text { Ref. Reference category }\end{array}$} \\
\hline
\end{tabular}

or no access to radio. Access to TV also has profound effect on the likelihood of health facility delivery as women who watch TV at least once a week are 6.97 times more likely (95\% CI 5.903 8.236; $\mathrm{p}<.001$ ) to opt for health facility delivery than the women who watch TV less than once a week or don not watch TV at all (Table 4). 
Overall, the analysis results confirm that media habits in terms of frequency of reading Newspaper, listening to radio and watching TV individually does show significant effect on health facility delivery practices. In addition, women who have habits of using all three types of communication media are 7 times more likely (95\% CI 4.334-12.273) to have health facility delivery than those who do not use different media channels (Table 4).

\subsection{Economic factors and utilization of health facility delivery services in Nepal}

Regarding economic characteristics, a high percentage of women who were working service/trade sector $(65.4 \%)$ had their last delivery at a health institution. The second highest percentage of women (56.3\%) having health facility delivery were currently not working. Relatively high percentages of women working in manual labor sector $(47.8 \%)$ had also delivered last pregnancy at health institution. Similarly, more than half of women $(54.6 \%)$ whose husband was in service/trade sector also had health facility delivery (Table 5).

\begin{tabular}{|c|c|c|c|c|c|c|}
\hline \multirow{2}{*}{ Description } & \multicolumn{2}{|c|}{ Health facility delivery } & \multicolumn{2}{|c|}{$\begin{array}{c}\text { Delivery at home or } \\
\text { other places }\end{array}$} & \multicolumn{2}{|c|}{ Total respondents } \\
\hline & Number & Percent & Number & Percent & Number & Percent \\
\hline \multicolumn{7}{|l|}{$\begin{array}{l}\text { Respondent's } \\
\text { Occupation* }\end{array}$} \\
\hline Manual & 87 & 47.8 & 93 & 51.1 & 182 & 100.0 \\
\hline Service/trade & 261 & 65.4 & 133 & 33.3 & 399 & 100.0 \\
\hline Agriculture & 599 & 24.8 & 1775 & 73.5 & 2415 & 100.0 \\
\hline Not working & 647 & 56.3 & 480 & 41.7 & 1150 & 100.0 \\
\hline Total & 1597 & 38.5 & 2481 & 59.8 & 4149 & 100.0 \\
\hline \multicolumn{7}{|l|}{$\begin{array}{l}\text { Husband's } \\
\text { Occupation* }\end{array}$} \\
\hline Manual & 433 & 32.9 & 855 & 65.0 & 1315 & 100.0 \\
\hline Service/trade & 922 & 54.6 & 749 & 44.4 & 1688 & 100.0 \\
\hline Agriculture & 195 & 19.4 & 792 & 78.8 & 1005 & 100.0 \\
\hline \multicolumn{7}{|l|}{ Not working } \\
\hline & 1597 & 38.5 & 2481 & 59.8 & 4149 & 100.0 \\
\hline \multicolumn{7}{|l|}{$\begin{array}{l}\text { Respondent's } \\
\text { Employment* }\end{array}$} \\
\hline Yes & 715 & 30.7 & 1577 & 67.7 & 2329 & 100.0 \\
\hline \multirow[t]{2}{*}{ No } & 883 & 48.5 & 903 & 49.6 & 1820 & 100.0 \\
\hline & 1598 & 38.5 & 2480 & 59.8 & 4149 & 100.0 \\
\hline \multicolumn{7}{|l|}{$\begin{array}{l}\text { Household Wealth } \\
\text { Status* }\end{array}$} \\
\hline Rich & 916 & 66.3 & 449 & 32.5 & 1382 & 100.0 \\
\hline Middle & 468 & 33.9 & 898 & 65.0 & 1382 & 100.0 \\
\hline Poor & 213 & 15.4 & 1133 & 81.9 & 1383 & 100.0 \\
\hline Total & 1597 & 38.5 & 2480 & 59.8 & 4147 & 100.0 \\
\hline \multicolumn{7}{|c|}{$\begin{array}{l}\text { Source: Calculated from NDHS } 2011 \text { data files } \\
\text { Weights are applied } \\
\text { *71 missing cases } \\
\text { Ref: Reference category }\end{array}$} \\
\hline
\end{tabular}

The second highest percentage of women (32.9\%) having health facility delivery were those whose husband was manual worker. Likewise, nearly half of women $(48.5 \%)$ who were not employed at the time of survey also had health facility delivery. By wealth index, two-third (66.3\%) from rich and about one-third (33.9\%) women from middle wealth groups had health facility delivery of their last child. Only 15 percent women from poor group had opted for health facility delivery of their last child (Table 5). 
Husband occupation has significant effect on health facility delivery as the analysis result shows that women whose husband's occupation is in service/trade sector are 5 times more likely (95\% CI 3.9826.254) to opt for health facility delivery than those women whose husband's occupation is in other sectors. Similarly, household wealth has significant effect on health facility delivery as the logistic regression analysis shows that women from rich household are more than 10 times likely (95\% CI 8.746-13.474) and middle wealth households more than twice likely (95\% CI 2.232-3.439) to have health facility delivery than the women from poor households (Table 6).

\begin{tabular}{|c|c|c|c|}
\hline Description & Odds ratio (OR) & $\begin{array}{c}\text { Confidence } \\
\text { interval (CI) }\end{array}$ & $P$ value \\
\hline \multicolumn{4}{|l|}{ Respondent's Occupation } \\
\hline Not working & .697 & $.469-1.034$ & 0.073 \\
\hline Agriculture & 1.460 & $1.085-1.965$ & 0.012 \\
\hline Service/trade & .250 & $.207-.303$ & 0.000 \\
\hline Manual & Ref. & & \\
\hline \multicolumn{4}{|l|}{ Husband's Occupation } \\
\hline Manual & 2.051 & $1.614-2.607$ & 0.000 \\
\hline Service/trade & 4.990 & $3.982-6.254$ & 0.000 \\
\hline Agriculture & Ref. & & \\
\hline \multicolumn{4}{|l|}{ Household Wealth Status } \\
\hline Rich & 10.855 & $8.746-13.474$ & 0.000 \\
\hline Middle & 2.770 & $2.232-3.439$ & 0.000 \\
\hline Poor & Ref. & & \\
\hline \multicolumn{4}{|c|}{ Respondent's employment } \\
\hline Yes & .463 & $.395-.543$ & 0.000 \\
\hline No & Ref. & & \\
\hline \multicolumn{4}{|c|}{$\begin{array}{l}\text { Source: Calculated from NDHS } 2011 \text { data files } \\
\text { Weights are applied } \\
\text { Ref: Reference category }\end{array}$} \\
\hline
\end{tabular}

\section{Concluding Remarks}

The analysis shows large variations and gaps in the utilization of health facility delivery services based on demographic, social and economic characteristics of women such as women's age, parity and age at $1^{\text {st }}$ childbirth, caste/ethnicity and levels of education, employment status, household wealth and many others.

The analysis results have indicated that demographic factors like age, parity, age at first birth and utilization of ANC services 4 or more times have significant effect on the utilization of health facility delivery in Nepal. Previously done studies in Ethiopia (Teferra, Alemu \& Woldeyohannes, 2012), Nigeria (Dahiru \& Oche, 2015) and Nepal (Dahal, 2013) have found strong effect of the demographic factors on health facility delivery. Similarly, the analysis result also showed strong effect of social factors like ethnicity, place of residence, level of education and use of media channels -newspaper, radio, and TV on health facility delivery practices of women. Studies conducted in Nepal and other developing countries have also clearly exhibited the influence of social variables on use or non-use of health facility for delivery of births (Nzioki, et.al, 2015; Dahal, 2013; Shah et al., 2015; and Dhak, 2013).

Economic factors also have strong effect in health facility delivery. Husband occupation is found to have significant effect on health facility delivery as the analysis result shows that women whose husband's occupation is in service/trade sector are 5 times more likely (95\% CI 3.982-6.254) to opt for health facility delivery than those women whose husband's occupation is in other sectors. Similarly, Household wealth has significant effect on health facility delivery as the logistic regression analysis shows that women from rich household are more than 10 times likely (95\% CI 8.746-13.474) 
and middle wealth households more than twice likely (95\% CI 2.232-3.439) to have health facility delivery than the women from poor households (Table 6). Similar effect has been found in a study conducted in India (Nair, et al., 2012) and Ethiopia (Mehari, 2013; Mehari and Wencheko, 2013) and Nepal (Shah et al., 2015).

Based on the finding of this analysis the study concludes that much effort should be made by the government to encourage more pregnant women to have health facility delivery. It is therefore recommended for the implementation of appropriate policy and program measures to address the existing variations and gaps in health facility delivery of births by women. Moreover, further in-depth research, both qualitative and quantitative, that address the demographic, social and economic diversity are recommended to be implemented which could help in understanding people's knowledge, attitudes, and practices on health facility delivery care. These researches also need to focus on the existing barriers in accessing health facility delivery services in Nepal, especially among the women who are disadvantaged and marginalized.

\section{References}

Bloom, S. S. \& Lippeveld, T. (1999). Does antenatal care make a difference to safe delivery? (Research Study Report in India). Urban Uttar Pradesh: Health Policy and Planning. Retrieved From http://www.researchgate.net/publication/12948370.

Dahal, R. K. (2013). Factors influencing the choice of place of delivery among women in eastern rural Nepal. International Journal of Maternal and Child Health. Retrieved From http://www.academia.edu/8744603/.

Dahiru, T. \& Oche, M. O. (2015). Determinants of antenatal care, institutional delivery and postnatal care services utilization in Nigeria. The Pan African Medical Journal, 23. Retrieved From http://www.panafrican-med- journal.com/content/article/21/321/ full/\#. Vqc581JJhdi.

Dhak, B. (2013). Use of maternal health care in rural India: Relative importance of socio-economic status and accessibility. Journal of Population and Social Studies, 21 (2). Retrieved From https://www.Journal+of+Population+and+Social+Studies $\quad \mathrm{C}+$ Volume+21+Number+2+ 28Supplement $29+$ July $+2013 \&$ ie $=$ utf $-8 \&$ oe $=$ utf -8 .

Mehari, A. M. (2013). Levels and determinants of use of institutional delivery care services among women of childbearing age in Ethiopia: Analysis of EDHS 2000 and 2005 data (DHS Working Papers). Maryland: ICF International. Retrieved From dhsprogram.com/publications/citations/cite_WP83.

Mehari, K. \& Wencheko, E. (2013). Factors affecting maternal health care services utilization in rural Ethiopia: A study based on the 2011 EDHS data, Ethiop. J. Health Dev, 27. Retrieved From www.ajol.info.

MoH, New ERA, and ORC Macro International Inc., (2002). Nepal Demographic and Health Survey 2001. Kathmandu: Ministry of Health and Population, New ERA, and Macro International Inc.

MoHP, New ERA, and Macro International Inc. (2007). Nepal demographic and health survey 2006. Kathmandu: Ministry of Health and Population, New ERA, and Macro International Inc.

MoHP, New ERA, and ICF International Inc. (2012). Nepal Demographic and Health Survey 2011. Kathmandu, Nepal: Ministry of Health and Population, New ERA, and ICF International, Calverton, Maryland.

MoH, New ERA, and ICF. (2017). Nepal Demographic and Health Survey 2016. Kathmandu,Nepal: Ministry of Health, New ERA, and ICF International, Calverton, Maryland.

Nair, M., Ariana P. \& Webster, P. (2012). What influences the decision to undergo institutional delivery by skilled birth attendants? A cohort study in rural Andhra Pradesh, India. The International Electrical Journal of Rural and Remote Health Research, Education, Practice. Retrieved From http://www.rrh.org.au/articles/ subviewasia.asp?ArticleID=2311.

Nziokil, J. M., Onyango, R. O. \& Ombaka, J. H. (2015). Socio-demographic factors influencing maternal and child health service utilization in Mwingi; A Rural Semi-Arid District in Kenya. American Journal of Public Health Research. Retrieved From www.sciepub.com/portal/downloads?doi=10.12691/ajphr. 
Shah, R., Rehfuess, E. A., Maskey. M. K., Fischer, R., Bhandari, P. B. \& Delius, M. (2015). Factors affecting institutional delivery in rural Chitwan district of Nepal: A community-based crosssectional study. BMC Pregnancy and Childbirth. Retrieved From www.biomedcentral.com/1471-2393/15/27

Suvedi, B. K., Pradhan, A., Barnett, S., Puri, M., Chitrakar, S. R., Poudel, P., Sharma, S. \& Hulton, L. (2009). Nepal maternal mortality and morbidity study 2008/2009: Summary of preliminary findings. Kathmandu: DoHS/Ministry of Health and Population.

Teferra, A. S., Alemu, F. M. \& Woldeyohannes, S. M. (2012). Institutional delivery service utilization and associated factors among mothers who gave birth in the last 12 months in Sekela District, North West of Ethiopia: A community based cross sectional study. BMC Pregnancy and Childbirth. Retrieved From http://www.biomedcentral.com/1471-2393/12/74

Tsegay, Y., Tesfay, G., Goicolea, I., Edin, K., Lemma, H. \& Sebastian, M. S. (2013). Determinants of antenatal and delivery care utilization in Tigray region, Ethiopia: A cross-sectional study. International Journal for Equity in Health. Retrieved From http://www.equityhealthj.com/content/12/1/30.

UNFPA (2004). Maternal mortality update 2004: Delivering into good hands. New York. Retrieved From http://www.unfpa.org/sites/default/files/pub-pdf/mmupdate 05_eng.pdf

WHO (2012). Trends in maternal mortality: 1990 to 2010. Geneva: WHO. Retrieved From http://apps.who.int/iris/bitstream/10665/44874/1/9789241503631 eng.pdf

Note: Views and opinions expressed in this article are the personal views of author 